\title{
Competitive positron and positronium trapping in porous media
}

\author{
Zs. Kajcsos ${ }^{a}$,*, L. Liszkay ${ }^{\mathrm{a}}$, G. Duplâtre ${ }^{\mathrm{b}}$, K. Lázár ${ }^{\mathrm{c}}$, L. Lohonyai ${ }^{\mathrm{a}}$, L. Varga $^{\mathrm{a}}$, \\ P.M. Gordo ${ }^{\mathrm{d}}$, A.P. de Lima ${ }^{\mathrm{d}}$, C. Lopes de Gil ${ }^{\mathrm{d}}$, M.F. Ferreira Marques ${ }^{\mathrm{e}}$,

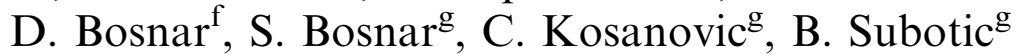 \\ ${ }^{a}$ KFKI Research Institute for Particle and Nuclear Physics, PO Box 49, H-1525 Budapest 114, Hungary \\ ${ }^{\mathrm{b}}$ Lab. Chim. Nucléaire, IReS, IN2P3/CNRS, BP. 28, 67037 Strasbourg Cedex 2, France \\ ${ }^{\mathrm{c}}$ Institute of Isotopes CRC HAS, PO Box 77, H-1525 Budapest, Hungary \\ ${ }^{\mathrm{d}}$ Department of Physics, University of Coimbra, 3004-516 Coimbra, Portugal

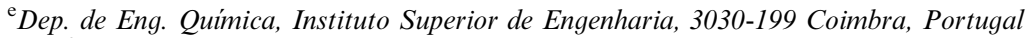 \\ ${ }^{\mathrm{f}}$ Department of Physics, University of Zagreb, Bijenicka 32, Zagreb, Croatia \\ ${ }^{\mathrm{g}}$ Division of Mater. Chem., Ruder Boskovic Institute, Bijenicka 54, PO Box 180, Zagreb, Croatia
}

\begin{abstract}
Positron annihilation acquired acceptance for structural investigation of solids but results in porous media - where positron lifetime spectroscopy (LT) reveals substantial Ps formation - were ambiguous. Data on zeolites lead to the conclusion that Ps trapping in competing "extended free volume" sites, inhomogeneous regions and grain boundaries occurs. Furthermore, positron trapping must also be considered. Systematic errors due to incomplete time range selection are discussed, significance and importance of corrections for $3 \gamma / 2 \gamma$ counting efficiency differences are shown in practice.
\end{abstract}

(C) 2006 Elsevier Ltd. All rights reserved.

Keywords: Positron annihilation in zeolites; Positronium trapping; Long-living $o$-Ps states

\section{Introduction}

Structural studies of solids employ positron annihilation techniques (PAT) as routine tools but in highly inhomogeneous systems and porous media the PAT data show a complexity which does not facilitate easy interpretation. Positron lifetime (LT) measurements reported for fine powders (Paulin and Ambrosino, 1968) reveal the existence of annihilation modes with long lifetime components associated with the annihila-

\footnotetext{
*Corresponding author. Fax: + 3613959151 .

E-mail address: kajcsos@rmki.kfki.hu (Zs. Kajcsos).
}

tion of ortho-positronium (o-Ps) in various free or confined states. Apart from the basic scientific interest directed to understand the origin of those lifetime components it became soon clear that ascribing those long components to trapped $o$-Ps states may supply means to identify and characterize the respective structural trapping sites. The simple quantum-well model (Tao, 1972; Eldrup et al., 1981; Jean, 1990)correlating the $o$-Ps lifetime to the size of the trapping sites - was applied with noticeable success. This model has been developed further recently (e.g., Goworek et al., 2000), taking into account non-spherical geometry as well. By all those models, the radius $(R)$ of the free 
volumes (whether spherical, cylindrical or cuboid) is related to the resulting lifetime of the $o$-Ps, thus providing one with a unique method to determine nanoscale-size Ps trapping sites (i.e., intrinsic or extrastructural free volumes) in a range of $0.1-10 \mathrm{~nm}$. It is a positive development that ab initio calculations have already been started to calculate the free volumes (Bug et al., 2004) and estimation of lifetimes is being targeted also for cases when liquids are present in the voids (Bug et al., 2005).

One of the important terrains of those PAT applications is the study of zeolites (Debowska et al., 1988; Chen et al., 1995; Duplâtre et al., 1999; Kajcsos et al., 2001; Zaleski and Borowka, 2004). Their highly porous structure, large external and internal specific surface bring about properties of high practical value, e.g. in catalysis or in radioactive waste management as well, where also the information on the amount and distribution of "free volume sites" is of primary importance. The silicate skeleton of zeolites furnishes the ionization electrons (produced along the track of the slowing down $e^{+}$) and provides the free spaces, needed to accommodate the $o$-Ps formed. The long lifetime values and high relative abundance $(10-130 \mathrm{~ns}$, ca. 10-30\%, e.g., Kajcsos et al., 2001) denote a weak interaction of $o$-Ps with its surroundings. These siliceous solids comprise a variety of open spaces, either structural channels and cages, or extrinsic nano- and mesopores. The Ps states are sensitive to additives (e.g., surface-bound ions, gases), which shorten the $o$-Ps lifetime and may also influence the relative intensities. Whereas $o$-Ps decays in vacuum intrinsically by emitting three $\gamma$ rays, its extrinsic decay in matter is governed by interactions with electrons of the surrounding medium, and through a "pick-off" process resulting in a dominantly $2 \gamma$-decay. Similarly, for the long-living trapped $o$-Ps lifetime states $3 \gamma$-decay prevails which calls attention to the fact that the $3 \gamma$-contribution to the annihilation pattern will be high, whereas a shortening of these components due to interactions with new agents leads mostly to $2 \gamma$-annihilation. Quantitative information on the relative concentration of various kinds of trapping centres could be obtained through the intensity of the respective (trapped) $o$-Ps-species, as is the case for trapped positrons in defect studies of solids. As, however, the overall counting efficiencies may drastically differ for the $3 \gamma$-and $2 \gamma$-decay modes leading to a distortion in the measured intensities of the various positron and Ps states, serious doubts are cast on the relevance of the measured $o$-Ps intensity with respect to the true, physical intensity. Thus, the determination of the true LT intensities requires that the ratio $\rho$ of the $3 \gamma /$ $2 \gamma$ counting efficiencies be determined (e.g., Dauwe, 1992; Kajcsos et al., 1995; Kajcsos et al., 2005), which might more easily be achieved when combining LT and Doppler broadening (DB) measurements.
With the intention of better understanding the main processes influencing the fate of positrons in porous systems, we extended our work to further questions of methodology and to those of the evaluation techniques and here we also reconsider and revisit some of our earlier data on the basis of newer insight gained since. Reference to our preliminary results (reported separately by Bosnar et al., 2006) obtained with LT measurements on the progress of crystallization of zeolites out of their amorphous precursors will also be made.

\section{Experimental}

Zeolite samples were studied by PAT with the aim of characterizing various forms of free volume present. In the present short work we shall refer only to some selected results but a comprehensive experimental outline is necessary for the data interpretation. Host matrices with known high Ps yield (Duplâtre et al., 1999; Kajcsos et al., 2000; Liszkay et al., 2001) were selected (MFI silicalite-1, fajausite, Na-Y, Na-X, etc.), taking well-documented samples, characterized by X-ray and thermogravimetry measurements as well. These siliceous solids comprise a variety of open spaces, either structural channels and cages, or extrinsic nano- and mesopores. Each of these sites, with their respectively different sizes and shapes, can give rise to a specific $o$-Ps component, the experimental difficulty lies "only" with recording the spectra with high enough statistics.

The non-framework cations are located in channels and voids and are coordinated with water molecules inside the channel. The cation-size influences the size of its hydration shell and the pore volume occupancy by water molecules. The removal of water-necessary to make the free volume available to $o$-Ps - was achieved by appropriate vacuum heat treatment (Liszkay et al., 2001).

LT measurements were performed on samples prepared by pressing (with $0.3 \mathrm{GPa}$ ) the zeolite powders into $1-1.5 \mathrm{~mm}$ thick disks of $8-13 \mathrm{~mm}$ diameter, sandwiching the ${ }^{22} \mathrm{Na}$ source $(0.5-2.0 \mathrm{MBq}$ activity, sealed between thin Kapton or Ni foils) between two sample disks, wrapped in Al-foil. LT measurements were performed on the as-prepared sample assemblies, which were placed into a glass holder, evacuated or in a part of the experiment filled with high purity $\mathrm{N}_{2}$ gas at atmospheric pressures (Kajcsos et al., 2000). For performing temperature-dependent measurements in the range of $110-300 \mathrm{~K}$, we had a sample-holder cryostat operating with gas cooling from a liquid nitrogen reservoir. Earlier results (Kajcsos et al., 2000, 2005) have shown that the capillary gas condensation at low temperatures practically eliminates the $3 \gamma$-decaying long-living $o$-Ps components and could allow so to 
determine the ratio $\rho$ of the $3 \gamma / 2 \gamma$ counting efficiencies which we want to apply for our data.

Two fast-fast coincidence LT spectrometers were employed, one featuring a pair of XP 2020 URQ photomultipliers equipped with $\mathrm{BaF}_{2}$ scintillators, whereas the other working with XP 2020 photomultipliers equipped with NE111 plastic scintillators. The wide energy selection windows $(50 \%$ on the start and $80 \%$ on the stop line) enabled high efficiency recording of $3 \gamma$-events, still with a 250 ps FWHM time resolution for ${ }^{60} \mathrm{Co}$ with the $\mathrm{BaF}_{2}$ scintillators and 290 ps FWHM for the plastic scintillators. The LT spectra were recorded employing simultaneously two time-to-pulse height converter units, one set to a "short" time range of $100 \mathrm{~ns}$ whereas the other to a "long" time range of $2 \mu \mathrm{s}$. The output time spectra were recorded for the $100 \mathrm{~ns}$ setting in a Microfast $16 \mathrm{k}$ PC MCA, whereas for the $2 \mu$ s setting the time-to-pulse-height-converter output signal was split further into two branches, one led into a PC-based 4k MCA and the other one into a Microfast $16 \mathrm{k}$ PC MCA with the intention to estimate the distorting influence of the compliance of time range setting with the range of lifetimes and also to compare the performance of two fully differing MCA designs. The time calibration values for the various settings were of $200.3,40.1$ and $99.8 \mathrm{ps} / \mathrm{ch}$, respectively. Integral counts of at least $2 \times 10^{6}-1 \times 10^{7}$ were collected under the various conditions. The reproducibility of the spectra was found good. The lifetimes and relative intensities were evaluated (with appropriate source corrections) using the LT v.9 fitting program (Kansy, 1996).

For the independent observation of the $3 \gamma / 2 \gamma$ fraction, full $\gamma$-energy —or extended Doppler (XDB) - measurements were performed (energy range: $40 \mathrm{keV}-1.4 \mathrm{MeV}$ ) using a Canberra HPGe detector with $1.86 \mathrm{keV}$ FWHM resolution at the $1.28 \mathrm{MeV} \gamma$ line of the $1 \mathrm{MBq}{ }^{22} \mathrm{Na}$ positron source. The XDB spectra were recorded in a Microfast $16 \mathrm{k}$ analyser. The $1.28 \mathrm{MeV}$ photopeak was used to monitor the number of positrons, enabling to trace alterations between the $2 \gamma$ - and $3 \gamma$-channels. Changes in the relative counts of the annihilation peak are proportional with the number of positrons leaving the $2 \gamma$-annihilation channel and annihilating via $3 \gamma$ decay. As a "pure" $2 \gamma$-annihilation case, spectra measured on water-saturated pellets were used (the negligible presence of $3 \gamma$ annihilation was proven by lifetime measurements). Details for the corrections were given by Liszkay et al., 2001.

\section{Results and discussion}

A satisfactory fit of the LT spectra usually requires at least four lifetime components: $0.2-0.5 \mathrm{~ns}$ combining the $p$-Ps and $e^{+}$-annihilations, $1-4 \mathrm{~ns}$ for $o$-Ps probably trapped in microvoids of the lattice, $10-90 \mathrm{~ns}$ for $o$-Ps decaying in internal channels of the zeolites and $100-135 \mathrm{~ns}$ for $o$-Ps decaying outside of the crystallites, in the intergrain space (Kajcsos et al., 2001), whereas mesopores may also contribute to this latter two components (Kajcsos et al., 2005). It has been also shown already that even a higher number of lifetime components could be significantly resolved: for silicalite1 MFI samples five components were extracted. Furthermore, in case of LT measurements performed on the MFI samples in closed $\mathrm{N}_{2}$ gas atmosphere, at low temperatures due to the capillary condensation into the channels and cages of the zeolite skeleton a newer component-ascribed to a Ps-bubble-like state in the condensed gas-became evident (Kajcsos et al., 2001, 2005), as will be discussed further.

The intensities of the long-lived components are subject to corrections due distortions by the unequal efficiencies for the detection of $2 \gamma$ - and $3 \gamma$-events (Kajcsos et al., 2005), and the registered spectra mirror the "survival proportions" of the various components and not those of the "birth fractions" (Kajcsos et al., 2001; Liszkay et al., 2001). The smaller the trapping site in which the $o$-Ps is confined, the higher the $2 \gamma$-decay fraction, whereas the larger the site, the higher the $3 \gamma$ decay fraction will be. Interestingly, preliminary studies on the gel-preparation technique have shown that partially exchanged gel samples exhibit higher contribution of long-living components as the gel sample exchanged in a higher extent, i.e., latent crystallization centres may induce unexpectedly high fraction of Ps. This line of studies will be continued in the future.

As an example for the differences encountered for the actual values evaluated for two different silicalite-1 sample featuring very long lifetimes, the results obtained from spectra (recorded with the "long range" setting) without correction for the efficiency differences for the $2 \gamma$-decay and the $3 \gamma$-decay recording, respectively, and with correction for these differences are presented in Table 1. (The values of $\rho$ were determined comparing LT and DB measurements at room- and low temperatures, where the $3 \gamma$-fraction becomes negligible.) Obviously, the influence of a higher $\rho$ value is more pronounced on the intensities, and it is also obvious that the corrections for the actual "birth population" relative intensities modify the intensity values of the short components as well. It is also clear that in case of $\rho=1$ no corrections are necessary. As the actual value of $\rho$-for given detectors-depends mostly on the energy window settings and on the detector solid angles, one can aim at approximating the value $\rho=1$, what we did in most cases, being aware of the fact that one can gain simplicity in the evaluation procedure on this way at the expense of efficiency and statistics.

The two LT spectrometers with NE111 and $\mathrm{BaF}_{2}$ scintillators, respectively, gave a reassuring equivalency 
Table 1

Lifetimes and relative intensities for two MFI samples (sample $A$ : grain size: $10 \mu \mathrm{m}$, sample $B$ : grain size: $12 \mu \mathrm{m}$ ), as evaluated uncorrected and corrected with the respective $\rho$ values, which were determined comparing room temperature and low temperature LT spectra (Kajcsos et al., 2005)

\begin{tabular}{|c|c|c|c|c|c|c|c|c|c|c|c|}
\hline Samples & $\begin{array}{l}\tau_{1}(\mathrm{~ns}) \\
( \pm 0.1)\end{array}$ & $\begin{array}{l}\tau_{2}(\mathrm{~ns}) \\
( \pm 0.1)\end{array}$ & $\begin{array}{l}\tau_{3}(\mathrm{~ns}) \\
( \pm 0.2)\end{array}$ & $\begin{array}{l}\tau_{4}(\mathrm{~ns}) \\
( \pm 1.2)\end{array}$ & $\begin{array}{l}\tau_{5}(\mathrm{~ns}) \\
( \pm 1.6)\end{array}$ & $\begin{array}{l}I_{1}(\%) \\
( \pm 0.6)\end{array}$ & $\begin{array}{l}I_{2}(\%) \\
( \pm 0.3)\end{array}$ & $\begin{array}{l}I_{3}(\%) \\
( \pm 0.4)\end{array}$ & $\begin{array}{l}I_{4}(\%) \\
( \pm 0.4)\end{array}$ & $\begin{array}{l}I_{5}(\%) \\
( \pm 0.3)\end{array}$ & $\rho$ \\
\hline$A$ (uncorr.) & 0.35 & 2.05 & 5.60 & 35.1 & 135.4 & 42.7 & 24.1 & 2.6 & 2.5 & 28.1 & \\
\hline$A$ (corr.) & 0.35 & 2.05 & 5.60 & 35.1 & 135.4 & 49.0 & 27.2 & 2.9 & 2.4 & 18.5 & 1.8 \\
\hline$B$ (uncorr.) & 0.35 & 2.24 & 7.50 & 33.2 & 123.3 & 53.9 & 5.5 & 10.8 & 8.6 & 21.2 & \\
\hline$B$ (corr.) & 0.35 & 2.24 & 7.50 & 33.2 & 123.3 & 57.4 & 5.8 & 11.0 & 8.4 & 17.4 & 1.3 \\
\hline
\end{tabular}

The lifetimes are not influenced by the correction. (The errors on the intensities are given in absolute numbers.)

for the recorded spectra in the range of source activities used here, no distorting effects due to higher count rates on the system with the $\mathrm{BaF}_{2}$ scintillators were observed with the source activity employed here. The LT spectra with the $2 \mu$ s time range setting recorded with the two different types of MCA show an excellent agreement, a reassuring result, indeed.

The comparison of LT spectra recorded for a Na-Y sample with "short" and "long" time range setting (Fig. 1) shows that the inclinations of the decay curves coincide well, but as in the case of the "short" setting the background level is not reached still at the end of the recording range, the higher channel content at this position is taken as an artificially higher background and the intensities determined differ for the two cases by several percents (Table 2), depending on the degree how the actual time ranges and lifetimes commeasure. It means that in addition to data influence from the sample synthesis and treatment (e.g., Kajcsos et al., 2001) and the changes in the $3 \gamma / 2 \gamma$-ratio (e.g., Kajcsos et al., 2005), the short (incomplete) time range settings may also result in somewhat incorrect intensity values if the background level is not properly reached.

The generally employed procedure to record the spectra consecutively with "short" and "long" time range setting may cause a systematic error if not only the lifetime values but also the relative intensities are assumed to be identical in the various recordings. Also, as for the evaluation of the short components a fine enough time/channel definition is needed, unconventionally long recording times are required. In accordance with the general rule of thumb, if the time range may accommodate 5-6 decay times of the actual long-living component, both the lifetimes and intensities could be trusted. If, however, only less than two half-lives are contained - which was often the case in early experiments, the lifetimes may carry substantially higher errors and the intensity values may differ drastically from the factual values, underlying the necessity of using long settings of the time-to-pulse heights converters and large-range MCAs for the recording of LT spectra. In

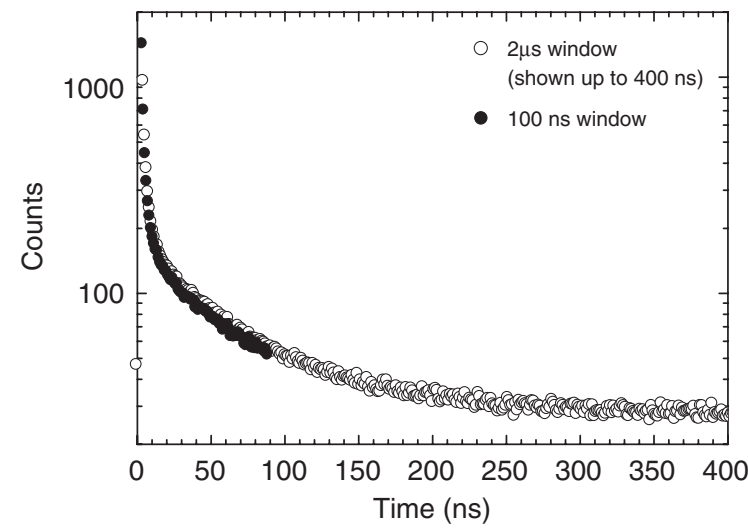

Fig. 1. The comparison of LT spectra recorded for a Na-Y sample with "short" and "long" time range setting shows that the inclinations of the decay curves coincide well, but the true background level is still not reached at the end of the recording range.

this case, the simultaneous evaluation of both the short and long-living components from the same data set becomes possible.

The free volume parameters defined from the TaoEldrup model give values as $R=8.7-13.7 \AA$. As, however, for the MFI samples only $\tau_{2}$ and $\tau_{3}$ could be correlated with crystalline free spaces (excluded volumes, intersections of channels, or the channels themselves, etc.,) and as there are no crystallographic formations present which could account for the very long lifetime values found for $\tau_{4}$ and $\tau_{5}$, these components probably correspond to $o$-Ps annihilating from mesopores, probably playing a role in other types of zeolites as well.

For the complexity of the picture the positrons bring on zeolites here we present the case when the silicalite-1 sample has been measured (with $\rho=1$ setting) in the presence of $\mathrm{N}_{2}$ in a broad temperature range. The lifetime values present one additional surprising contribution, becoming evident in the multi-component free 
Table 2

Lifetimes and relative intensities for two samples (sample $C$ : Na-X, sample $D$ : Na-X), as evaluated from the spectra recorded in the $2 \mu$ s and $100 \mathrm{~ns}$ time range, respectively

\begin{tabular}{|c|c|c|c|c|c|c|c|c|}
\hline Samples & $\begin{array}{l}\tau_{1}(\mathrm{~ns}) \\
( \pm 0.05)\end{array}$ & $\begin{array}{l}\tau_{2}(\mathrm{~ns}) \\
( \pm 0.07)\end{array}$ & $\begin{array}{l}\tau_{3}(\mathrm{~ns}) \\
( \pm 0.1)\end{array}$ & $\begin{array}{l}\tau_{4}(\mathrm{~ns}) \\
( \pm 0.6)\end{array}$ & $\begin{array}{l}I_{1}(\%) \\
( \pm 0.5)\end{array}$ & $\begin{array}{l}I_{2}(\%) \\
( \pm 0.2)\end{array}$ & $\begin{array}{l}I_{3}(\%) \\
( \pm 0.2)\end{array}$ & $\begin{array}{l}I_{4}(\%) \\
( \pm 0.3)\end{array}$ \\
\hline$C(2 \mu$ s range $)$ & 0.30 & 0.65 & 1.86 & 37.9 & 55.9 & 34.1 & 7.4 & 2.6 \\
\hline$C(100$ ns range $)$ & 0.25 & 0.61 & 1.90 & 35.1 & 56.9 & 34.4 & 7.3 & 1.4 \\
\hline$D(2 \mu$ s range $)$ & 0.31 & 0.65 & 1.87 & 63.2 & 52.2 & 30.1 & 8.7 & 9.0 \\
\hline$D(100 \mathrm{~ns}$ range $)$ & 0.30 & 0.66 & 5.60 & 35.1 & 56.7 & 30.5 & 8.4 & 5.4 \\
\hline
\end{tabular}

As $\rho=1$ value was set, no intensity corrections were needed. (The errors on the intensities are given in absolute numbers.)

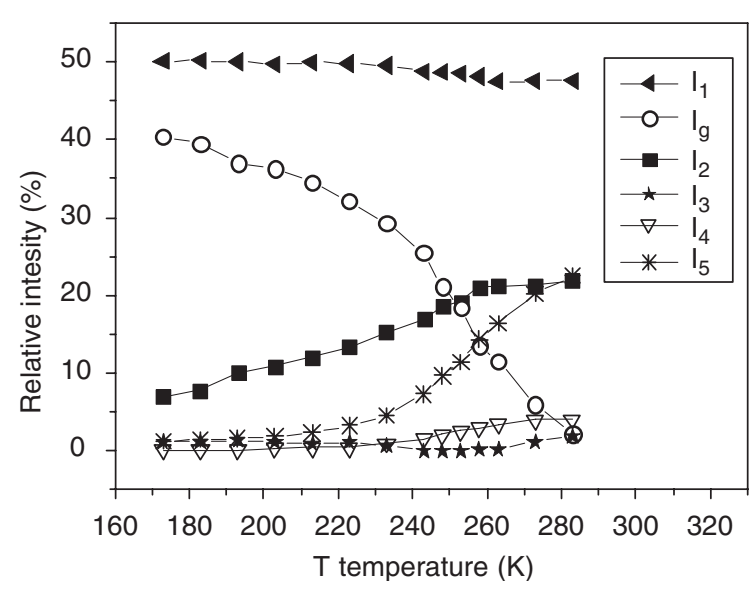

Fig. 2. The relative intensity values vs. temperature as measured for a MFI silicalite-1 sample in $\mathrm{N}_{2}$ gas at atmospheric pressure. $I_{g}$ denotes the intensity of the component connected with $o$-Ps annihilating in the gas. $I_{1}$ represents the "short lifetime" contribution, not resolved further into $p$-Ps, free positron or trapped positron sub-components.

evaluation of the data in the whole temperature range, successively re-fitting of the data after a stepwise identification of various basic components which were then better extracted in the "series fitting mode"-a most ingenious and useful feature in the LT v. 9 fitting code - are shown in Fig. 2. The additional component, $\tau_{g}=3.3 \mathrm{~ns}$ hardly visible at room temperature but becoming successively more and more dominant upon cooling could be identified with the annihilation of $o$-Ps in the adsorbed gas. Scrutinizing the intensities of the various components, it becomes obvious that in total, Ps-formation takes place in the whole temperature range at about $75 \%$ constant level, proving that this is a feature of the given zeolite structure. The $I_{1}$ component includes the contribution from $p$-Ps and free annihilation, and as $\tau_{1}$ substantially surpasses the $p$-Ps lifetime and bulk lifetime values for $\mathrm{SiO}_{2}$, a trapping of positrons could also be assumed.

\section{Conclusions}

The present results suggest a coherent picture for the positron behaviour in zeolites: a large fraction of the interacting $e^{+} / e^{-}$pairs initially created in the bulk (sometimes referred to as Ps, but not possessing lifetimes that exceed some $0.5 \mathrm{~ns}$ ) is rapidly expelled into the channels, in which it can develop into positronium, with long-living $o$-Ps state, too. The latter thereafter travels through the channels, rapidly losing its initial kinetic energy and sampling suitable structural free volumes or large defected sites, like mesopores as perspective trapping sites. It can reach the end of the channels only if it does not get trapped before. If trapped, Ps remains and decays in the trap with a lifetime depending on the size of this trap. The LT distribution in zeolites might be interpreted by the "free volume" trapping of $o$-Ps. Whereas the resulting lifetime might be correlated to the size of the trap, the saturation-near level of trapping may fully mask the proportionality between the trap concentration and the relative intensity of the respective lifetime component. The high level of trapping of $o$-Ps may shadow sensitivity to absolute structural features, too, showing great similarity to the behaviour of positrons in amorphous glasses where a saturation level trapping masks the possible influence of structural changes on the annihilation pattern. The high level of trapping, however, may allow the study of the relative changes of features of the internal walls by changes of the respective lifetimes upon e.g. ion decoration of the cages. To establish the true intensity ratios between various annihilation channels coming into existence in the complex structural pattern of micro- and nanoporous media, the combined application of the various PAT methods is necessary. Slow positron measurements should also be performed to clarify some questions mentioned before.

During the Ps-formation process, the influence of incomplete thermalization, delayed formation of Ps, diffusion of $o$-Ps before getting trapped, a distribution of lifetimes instead of discrete values, the possibility of 
transitions between various trapped states and that of the de-trapping should all be considered in details, just as well as trapping of positrons in porous media. It is also a challenge to provide experimental data for comparison with the recently started Path Integral Monte Carlo calculations of free volumes in porous materials.

\section{Acknowledgements}

These studies were first initiated in a HungarianFrench bilateral cooperation and were continued and supported later within a Hungarian Portuguese (P-23/ 03) and a Hungarian-Croatian (HR-1/2004) research agreement. Contributions through the research grants of the OTKA (Hungarian Research Fund, Grant nos. T029215, T030327, T032029, T032249, T046238, W15585, W15722) and in Croatia by the Ministry of Science, Education and Sports, contract nos. 0119259, A 679078 and 0098060 were essential.

\section{References}

Bosnar, S., Kosanović, C., Subotić, B., Bosnar, D., Kajcsos, Zs., Liszkay, L., Lohonyai, L., Molnár, B., Lázár, K., 2006. On the potential of positron lifetime spectroscopy for the study of early stages of zeolites formation from their amorphous precursors. Rad. Phys. Chem., in press, doi:10.1016/j.radphyschem.2006.03.045.

Bug, A.L.R., Muluneh, M., Waldman, J., Sterne, P.A., 2004. Positronium in solids: computer simulation of pick-off and self-annihilation. Mater. Sci. Forum 445\&446, 375-379.

Bug, A.L.R., Cronin, T.W., Sterne, P.A., Wolfson, Z.S., 2005. Simulation of positronium: toward more realistic models of void spaces in materials. Rad. Phys. Chem., in press, doi:10.1016/j.radphyschem.2006.03.043.

Chen, Z.Q., Tang, Z., Wang, S.J., 1995. Surface properties of zeolite characterized by positron annihilation technique. Mater. Sci. Forum 175-178, 667-670.

Dauwe, C., 1992. On the evaluation of the fraction of orthopositronium in lifetime spectra. Mater. Sci. Forum 105-110, 1857-1860.

Debowska, M., Abbé, J.C., Duplâtre, G., 1988. Positron annihilation studies in zeolites. Phys. Stat. Sol. B 146, 91-96.
Duplâtre, G., Kajcsos, Zs., Billard, I., Liszkay, L., Lázár, K., Lohonyai, L., Beyer, H.K., Caullet, P., Patarin, J., 1999. Free volumes in zeolites as seen by positrons. Stud. Surf. Sci. Catal. 125, 277-284.

Eldrup, M., Lightbody, D., Sherwood, J.N., 1981. The temperature dependence of positron lifetimes in solid pivalic acid. Chem. Phys. 63, 51-58.

Goworek, T., Ciesielski, K., Jasinska, B., Wawryszczuk, J., 2000. Temperature variations of average $o$-Ps lifetime in porous media. Radiat. Phys. Chem. 58, 719-722.

Jean, Y.C., 1990. Positron annihilation spectroscopy for chemical analysis: a novel probe for microstructural analysis of polymers. Microchem. J. 42, 72-102.

Kajcsos, Zs., Liszkay, L., Varga, L., Lázár, K., Brauer, G., Dauwe, C., 1995. The mixing of $2 \gamma$-and $3 \gamma$-events in the data collection of positron annihilation experiments: microcrystalline powders and zeolites. Mater. Sci. Forum 175-178, 959-962.

Kajcsos, Zs., Duplâtre, G., Liszkay, L., Billard, I., Bonnenfant, A., Azenha, E., Lázár, K., Pál-Borbély, G., Caullet, P., Patarin, J., Lohonyai, L., 2000. On the peculiarities of positron annihilation features in silicalite-1 and Y-zeolites. Rad. Phys. Chem. 58, 709-714.

Kajcsos, Zs., Duplâtre, G., Liszkay, L., Lázár, K., Lohonyai, L., Pál-Borbély, G., Beyer, H.K., Caullet, P., Patarin, J., 2001. Positrons and positronium in zeolites. Mater. Sci. Forum 363-365, 238-243.

Kajcsos, Zs., Liszkay, L., Duplâtre, G., Varga, L., Lohonyai, L., Pászti, F., Szilágyi, E., Lázár, K., Kótai, E., Pál-Borbély, G., Beyer, H.K., Caullet, P., Patarin, J., Azenha, M.E., Gordo, P.M., Lopes Gil, C., de Lima, A.P., FerreiraMarques, M.F., 2005. Positronium trapping in porous solids: means and limitations for structural studies. Acta Phys. Polon. A 107, 729-737.

Kansy, J., 1996. Microcomputer program for analysis of positron annihilation lifetime spectra. Nucl. Instr. Meth. Phys. Res. A 374 (2), 235-244.

Liszkay, L., Kajcsos, Zs., Duplâtre, G., Lázár, K., Pál-Borbély, G., Beyer, H.K., 2001. Positronium interactions in synthetic zeolites: effect of adsorbed water. Mater. Sci. Forum 363-365, 377-379.

Paulin, R., Ambrosino, G., 1968. Free annihilation of the ortho-positronium formed in some powders with a high specific surface. J. Phys. 29, 263-270.

Tao, S.J., 1972. Positronium annihilation in molecular substances. J. Chem. Phys. 56 (11), 5499-5510.

Zaleski, R., Borowka, A., 2004. Positron Study of MCM-41 Sieve Formation. Mater. Sci. Forum 445\&446, 364-366. 